\title{
Generation of Reverse Meniscus Flow by Applying An Electromagnetic Brake
}

\author{
ALEXANDER VAKHRUSHEV, ABDELLAH KHARICHA, \\ EBRAHIM KARIMI-SIBAKI, MENGHUAI WU, ANDREAS LUDWIG, \\ GERALD NITZL, YONG TANG, GERNOT HACKL, JOSEF WATZINGER, \\ and SVEN ECKERT
}

A numerical study is presented that deals with the flow in the mold of a continuous slab caster under the influence of a DC magnetic field (electromagnetic brakes (EMBrs)). The arrangement and geometry investigated here is based on a series of previous experimental studies carried out at the mini-LIMMCAST facility at the Helmholtz-Zentrum Dresden-Rossendorf (HZDR). The magnetic field models a ruler-type EMBr and is installed in the region of the ports of the submerged entry nozzle (SEN). The current article considers magnet field strengths up to $441 \mathrm{mT}$, corresponding to a Hartmann number of about 600 , and takes the electrical conductivity of the solidified shell into account. The numerical model of the turbulent flow under the applied magnetic field is implemented using the open-source CFD package OpenFOAM $\AA$. Our numerical results reveal that a growing magnitude of the applied magnetic field may cause a reversal of the flow direction at the meniscus surface, which is related the formation of a "multiroll" flow pattern in the mold. This phenomenon can be explained as a classical magnetohydrodynamics (MHD) effect: (1) the closure of the induced electric current results not primarily in a braking Lorentz force inside the jet but in an acceleration in regions of previously weak velocities, which initiates the formation of an opposite vortex (OV) close to the mean jet; (2) this vortex develops in size at the expense of the main vortex until it reaches the meniscus surface, where it becomes clearly visible. We also show that an acceleration of the meniscus flow must be expected when the applied magnetic field is smaller than a critical value. This acceleration is due to the transfer of kinetic energy from smaller turbulent structures into the mean flow. A further increase in the $\mathrm{EMBr}$ intensity leads to the expected damping of the mean flow and, consequently, to a reduction in the size of the upper roll. These investigations show that the Lorentz force cannot be reduced to a simple damping effect; depending on the field strength, its action is found to be topologically complex.

https://doi.org/10.1007/s11663-021-02247-x

(C) The Author(s) 2021

\section{INTRODUCTION}

ALEXANDER VAKHRUSHEV, ABDELLAH KHARICHA, and EBRAHIM KARIMI-SIBAKI are with the Christian Doppler Laboratory for Metallurgical Applications of Magnetohydrodynamics, Montantuniversität Leoben, Franz-JosefStrasse 18, 8700 Leoben, Austria. Contact e-mail: abdellah.kharicha@unileoben.ac.at MENGHUAI WU and ANDREAS LUDWIG are with the Chair of Simulation and Modeling of Metallurgical Processes, Montantuniversität Leoben, Franz-Josef-Strasse 18, 8700 Leoben, Austria. GERALD NITZL is with RHI Magnesita GmbH, Kranichberggasse 6, 1120 Vienna, Austria. YONG TANG and GERNOT HACKL are with the RHI Magnesita Technology Center, Magnesitstrasse 2, 8700 Leoben, Austria. JOSEF WATZINGER is with the Primetals Technologies Austria GmbH, Turmstrasse 44, 4031 Linz, Austria. SVEN ECKERT is with the Magnetohydrodynamics Department, Helmholtz-Zentrum Dresden-Rossendorf, Bautzner Landstraße 400, 01328 Dresden, Germany.

Manuscript submitted February 16, 2021; accepted June 7, 2021.

Article published online July 6, 2021.
ENSURING the quality of continuous cast (CC) products is becoming increasingly important in view of growing production rates. Uncontrolled fluid flow in the continuous casting mold is suspected of being responsible for various casting defects. Turbulent jet flow is an important phenomenon during the continuous casting process as the mold flow is mainly driven by the submerged jet emanating from the submerged entry nozzle (SEN). It influences the free surface stability, promotes superheat transport to the solidified shell as well as to the slag band, or poses the risk of introducing impurities and inclusions into the bulk of the slab. Electromagnetic brakes (EMBrs) are considered a powerful tool to provide an effective flow control. 
The striking influence of uniform transverse magnetic fields on liquid metal flows in ducts with various wall conductance ratios was observed in the early studies of Cuevas et al. ${ }^{[1,2]}$ The ability of DC magnetic fields to dampen fluctuations in highly turbulent flows is supposed to have attractive application potential for flow control in continuous casting, for example, to prevent undesired remelting of the solidified shell. ${ }^{[3-5]}$ However, the magnetic field effect is anisotropic and, thus, could become rather complex. Studies on mixed convection, which represents a superposition of buoyancy and forced convection, found both a stabilizing and a redistributing impact. ${ }^{[6]}$ There are already a couple of studies published that show, for the flow in the continuous casting mold, that the use of an EMBr does not exclusively result in a damping of the flow but also affects the flow structure in a way that can possibly lead to an acceleration and destabilization of the flow by large-scale fluctuations or to disturbances at the meniscus. $^{[6-10]}$

Systematic model experiments on laboratory scale in low melting point metal alloys were performed at Helmholtz-Zentrum Dresden-Rossendorf (HZDR) to study the effect of various externally applied magnetic fields on the flow inside a mockup of a conventional $\mathrm{CC}$ mold. ${ }^{[8,10-13]}$ The experimental setup was equipped with suitable measurement techniques to obtain quantitative data with high temporal and spatial resolution. In particular, new insights have been gained by varying the location of the EMBr system, as reported by Schurmann et al. ${ }^{[10]}$ A wide range of the complex phenomena in the metallurgical field can be investigated $a$ priori by means of numerical modeling, as reviewed by Thomas. ${ }^{[14]}$ Nowadays, a powerful strategy has been established that combines experimental work with extended numerical studies by different research groups. ${ }^{[9,12,15-17]}$ The numerical simulations effectively complement the parameter space that can be covered by the experiments and provide data at a high density and resolution. That is especially valuable for the industrial applications where the flow observations are limited to the meniscus region due to the harsh environment and high temperatures. On the other hand, the numerical simulations need to be validated by robust experimental data.

The application of electromagnetic fields for flow control in continuous casting requires a comprehensive understanding of the complex interactions. Improper application can also lead to unintended deterioration of the flow structure. For example, as recently reported by Schurmann et al., ${ }^{[13]}$ an electromagnetic stirrer has the potential to induce a desired flow structure, but under certain circumstances, it can also, on the contrary, lead to problems such as destabilization of the free surface. This study demonstrates that when choosing the magnetic field settings, the other process parameters, such as the different SEN types, must be considered carefully to achieve a beneficial result.

In a recent review, Cho and Thomas ${ }^{[18]}$ classified the influence of the applied magnetic field on the formation of different casting defects and suggested corresponding guidance for practical use of EMBrs.
Complementary to the studies mentioned here, the authors of this work have recently presented a very detailed numerical study of the induced electric current distribution during the EMBr process, focusing on the interaction with the turbulent flow and considering the effects of the presence of the solid shell, which is very important during real solidification in the $\mathrm{CC}$ process. ${ }^{[19]}$

A new freestanding adjustable combination $\mathrm{EMBr}$ type (FAC-EMBr) was numerically investigated in $\mathrm{Li}$ et $a l .{ }^{[20]}$ by varying the magnetic induction intensity, the SEN immersion depth, and the port angle for different casting speeds. Garcia-Hernandez et al. observed perturbations of the meniscus level in thin slab castings due to periodic flow alterations and refer to this turbulent behavior of the flow under the term dynamic distortions (DDs). ${ }^{[21]}$ The authors investigated whether and how the horizontal and vertical EMBrs can be applied to prevent the occurrence of DDs at the meniscus. While there seems to be some success in the case of the horizontal $\mathrm{EMBr}$, the authors surprisingly failed to show a way to prevent or control the DD phenomenon using the vertical EMBr. Recently, Vakhrushev et al. ${ }^{[22]}$ took both the viscoplastic behavior of the solidified shell and the magnetohydrodynamics (MHD) effects of the EMBr into account to simulate the turbulent flow and the shell thickness during the thin slab casting with and without the DC magnetic field.

The mini-LIMMCAST experimental setup at the HZDR is based on the geometries of industrial plants and uses conventional SEN types that are typical for practice in most casting mills. ${ }^{[10,11]}$ In these model experiments, it was found that the application of a horizontal magnetic field at Hartmann numbers of about 400 can also unintentionally accelerate and destabilize the meniscus flow in comparison to the situation without EMBr. Meanwhile, such a behavior was also reproduced by numerical simulations. ${ }^{[9,12,15,17,19]}$ However, in view of a couple of unanswered questions in this context, the authors are not yet aware of any further work specifically devoted to this phenomenon. Therefore, this study is devoted to numerical simulations considering the application of a horizontal ruler-type $\mathrm{EMBr}$ in a wide range of Hartmann numbers up to $600\left(\mathbf{B}_{\mathbf{0}}=0 \ldots 441 \mathrm{mT}\right)$. Our results reveal that the flow pattern dramatically changes with growing magnetic field strength. At a certain threshold value of the magnetic field, the formation of a "multiroll" structure is triggered, which is accompanied by an opposite flow direction at the meniscus. As the magnetic field grows, this flow pattern is consolidated and finally occupies the entire upper part of the CC mold.

Our parametric study based on wide and highly resolved magnetic field variations addresses the following main questions: (1) How does the flow structure change with the growing magnetic field strength? (2) What is the origin of initial meniscus acceleration and its later deceleration? (3) Which conditions and mechanisms are responsible for the formation of the opposite meniscus flow? 


\section{NUMERICAL MODEL}

In this section, a summary of the numerical model of the turbulent flow under the applied magnetic field is presented. The details of its in-house implementation using the open-source CFD package OpenFOAM ${ }^{\circledR} *[23]$

*OpenFOAM is distributed by the OpenFOAM Foundation and is freely available and open source, licensed under the GNU General Public License. The OpenFOAM Foundation Ltd, London, UK.

are described elsewhere. ${ }^{[19]}$

By including MHD (Lorentz) force $\mathbf{F}_{L}$ acting in the conducting melt under the applied constant magnetic field $\mathbf{B}_{0}$, the set of the incompressible Navier-Stokes equations becomes

$$
\nabla \bullet \mathbf{u}=0
$$

$$
\frac{\partial \mathbf{u}}{\partial t}+\nabla \bullet(\mathbf{u} \otimes \mathbf{u})=-\frac{1}{\rho} \nabla p+\nabla \bullet \tau_{\mathrm{lam}}-\nabla \bullet \tau_{\mathrm{SGS}}+\frac{1}{\rho} \mathbf{F}_{L}
$$

where $\mathbf{u}$ is the melt velocity; $\rho$ is the liquid density; $p$ is a pressure field; and $\tau_{\mathrm{lam}}$ and $\tau_{\mathrm{SGS}}$ are the laminar and the subgrid scale (SGS) Reynolds stress tensors, respectively.

Chaudhary et al. ${ }^{[9]}$ showed, that the wall-adapting local eddy-viscosity (WALE) turbulence model ${ }^{[24]}$ gives a better prediction of the turbulent flow than the standard Smagorinsky (SM) model ${ }^{[25]}$ based on the measurements in the mini-LIMMCAST experiment. The better performance of the WALE SGS model was recently confirmed by further numerical study of the same experimental setup including EMBr. ${ }^{[26]}$ Thus, the WALE turbulence model is used in the present work to simulate $\tau_{\mathrm{SGS}}$ : It is robust for complex geometries with strong mesh refinements, and it is capable of predicting the formation of coherent structures that can exist under the influence of the applied magnetic field. ${ }^{[9,24,27]}$

Since the magnetic Reynolds number is low for the CC applications $(\mathcal{R} m \ll 1),{ }^{[19]}$ the Maxwell's equations are reduced using the electric potential method. ${ }^{[28]}$ The induced current density $\mathbf{j}$ is given by the Ohm's law as follows:

$$
\mathbf{j}=\sigma \cdot\left(-\nabla \varphi+\mathbf{u} \times \mathbf{B}_{0}\right)
$$

where $\varphi$ is the electric potential and $\sigma$ is the electrical conductivity of the solid or liquid steel, respectively. From the charge conservation law $(\nabla \bullet \mathbf{j}=0)$, a Poisson equation is constructed for the electric potential,

$$
\nabla \bullet(\sigma \nabla \varphi)=\nabla \bullet\left(\sigma \cdot\left(\mathbf{u} \times \mathbf{B}_{0}\right)\right)
$$

and the corresponding Lorentz force is calculated as

$$
\mathbf{F}_{L}=\mathbf{j} \times \mathbf{B}_{0}
$$

The computational domain contains both liquid and solid regions: The outer boundaries of the liquid domain are electrically insulated; a thin layer of the highly conductive solid, attached to the mold walls, mimics the presence of the shell in the real continuous casting. The analysis of the solid conductance ratio, as well as the description and verification of the coupling algorithm, are presented elsewhere. ${ }^{[19]}$

\section{MODEL APPLICATION}

The present numerical model is applied to the mini-LIMMCAST setup equipped with a $\mathrm{CC}$ mold of a cross section of $140 \times 35 \mathrm{~mm}^{2}$ (Figure 1) and a ruler-type electromagnetic brake. ${ }^{[8,11,12]}$ The liquid Ga68In20Sn12 alloy was used in the experiment. The thermophysical melt properties were reported by Plevachuk et al. ${ }^{[29]}$

Initial simulations and the analysis of the meniscus velocity growth are done for the $\mathrm{EMBr}$ case positioned at the SEN bottom $92 \mathrm{~mm}$ below meniscus level; the peak value of the magnetic flux density is $312 \mathrm{mT}$. All studies are performed for the casting speed $u_{\text {pull }}=1.35 \mathrm{~m} / \mathrm{min}$, which relates to the SEN inlet velocity of $1.4 \mathrm{~m} / \mathrm{s}$. This specific configuration corresponds to the one reported in Thomas et al. ${ }^{[12]}$ The simulated geometry and the distribution of the applied magnetic field $\mathbf{B}_{0}$ are presented in Figures 1(a) and (b). The mold and the SEN walls are electrically insulating. It must be considered that the induced electric current can close in the solidified shell, which has a higher electrical conductivity than the liquid steel. In the experiments, 0.5 - $\mathrm{mm}$-thick brass plates are attached to the wide faces inside the mold to reflect the presence of the solid shell by matching the corresponding wall conductance ratio. ${ }^{[8]}$

According to the experimental setup, ${ }^{[1]}$ a CAD model and the numerical grid were constructed using the open-source package SALOME ${ }^{[30]}$ and snappyHexMesh OpenFOAM ${ }^{\circledR}$.

The details of the hex-dominant mesh can be seen in Figure 1(c). The mesh refinement close to the side walls is necessary to resolve the viscous and electromagnetic boundary layers. MHD boundary layers can be defined based on the Hartmann number $\mathcal{H a}$ :

$$
\mathcal{H} a=\left|\mathbf{B}_{0}\right| \cdot L_{0} \cdot \sqrt{\sigma_{\mathrm{liq}} / \rho \cdot \eta}
$$

where $L_{0}$ stands for the domain's length scale, which corresponds to the half-size of the mold along magnetic field lines. ${ }^{[31]}$ Electrical conductivity and kinematic viscosity of the fluid are expressed by the symbols $\sigma_{\text {liq }}$ and $\eta$, respectively.

The Hartmann magnetic boundary layer with the thickness $\Delta_{\mathrm{Ha}}$ exists on the walls perpendicular to the magnetic field. The Shercliff layer of size $\Delta_{\mathrm{Sh}}$ is formed at the parallel walls. ${ }^{[32]}$ The thicknesses of these layers can be estimated as follows: ${ }^{[31]}$

$$
\Delta_{\mathrm{Ha}} \cong L_{0} \cdot \mathcal{H} a^{-1}
$$

$$
\Delta_{\mathrm{Sh}} \cong L_{0} \cdot \mathcal{H} a^{-1 / 2}
$$


In the current study, the Hartmann number is $\mathcal{H} a \approx$ 417 for the reference magnetic field value of $312 \mathrm{mT}$. Thus, the Hartmann layer becomes $\Delta_{\mathrm{Ha}} \approx 50 \mu \mathrm{m}$ and the Shercliff layer is, correspondingly, $\Delta_{\mathrm{Sh}} \approx 1 \mathrm{~mm}$.

The wall conductance ratio,

$$
C_{\mathrm{wall}}=\frac{\delta_{\mathrm{wall}} \cdot \sigma_{\mathrm{sol}}}{L_{0} \cdot \sigma_{\mathrm{liq}}}
$$

of the attached brass plates with the thickness $\delta_{\text {wall }}=0.5 \mathrm{~mm}$ is sufficiently high $\left(C_{\text {wall }}=0.134\right)$ that a noteworthy part of the induced current closes in the solid wall. ${ }^{[8,12,19]}$

Additionally, the transport of the induced current occurs in the Shercliff boundary layer. ${ }^{[31]}$ No massive mesh refinement is required in the liquid bulk region, as discussed by the authors previously. ${ }^{[19]}$

Based on the casting speed, the simulation results are averaged through the time interval of 39 seconds. ${ }^{[19]}$ The second-order space integration of the gradient and advective schemes is used. The second-order backward time integration scheme was performed with an integration step of $5 \times 10^{-5} \mathrm{~s}$ to achieve a Courant number of $\mathrm{Co} \approx 0.15$. Hereafter, the time averaged velocity fields are presented and analyzed. For the induced current density distribution and for the interaction with the turbulent structures, the instantaneous results are used.

Before proceeding to the main studies of the present work, the numerical results were verified based on the published experimental and simulated data. ${ }^{[8,9,11,12]}$ In Figure 2, the comparison is shown for the flow without EMBr (Figure 2(a)) and with the applied magnetic field (Figure 2(b)). The distribution of the horizontal velocity component $u_{x}$ at the corresponding locations between the mold narrow face (NF) and the SEN showed good agreement both with the UDV measurements and with the modeled results. ${ }^{[8,9,11,12]}$

To start, the features of the simulated melt flow and the induced current behavior for the standard experimental setup performed at the HZDR GaInSn experiment are discussed. The mean velocity fields for the case without magnetic field and with the default value of $312 \mathrm{mT}$ are compared, as shown in Figure 3. Due to the strong turbulence in the flow, the mean field is quite smeared for the no EMBr case (Figure 3(a)). However, the jet region is clearly defined after the melt exits the SEN ports.

In the presence of the $\mathrm{EMBr}$, both strong upward and downward flows develop along the narrow (insulated) walls and along the meniscus surface, as shown in Figure 3(b).

The following observations are made in Figure 3: In the EMBr case (Figure 2(b)), the flow at the meniscus accelerates and the flow structure, shown by white arrows, significantly changes in the bulk. The flow in the lower mold region transforms from a strong recirculation zone to a plug-type downward flow due to the Lorentz force action. Furthermore, there is a tendency for the upper roll in Figure 2(a) to be split by a newly formed countervortex.

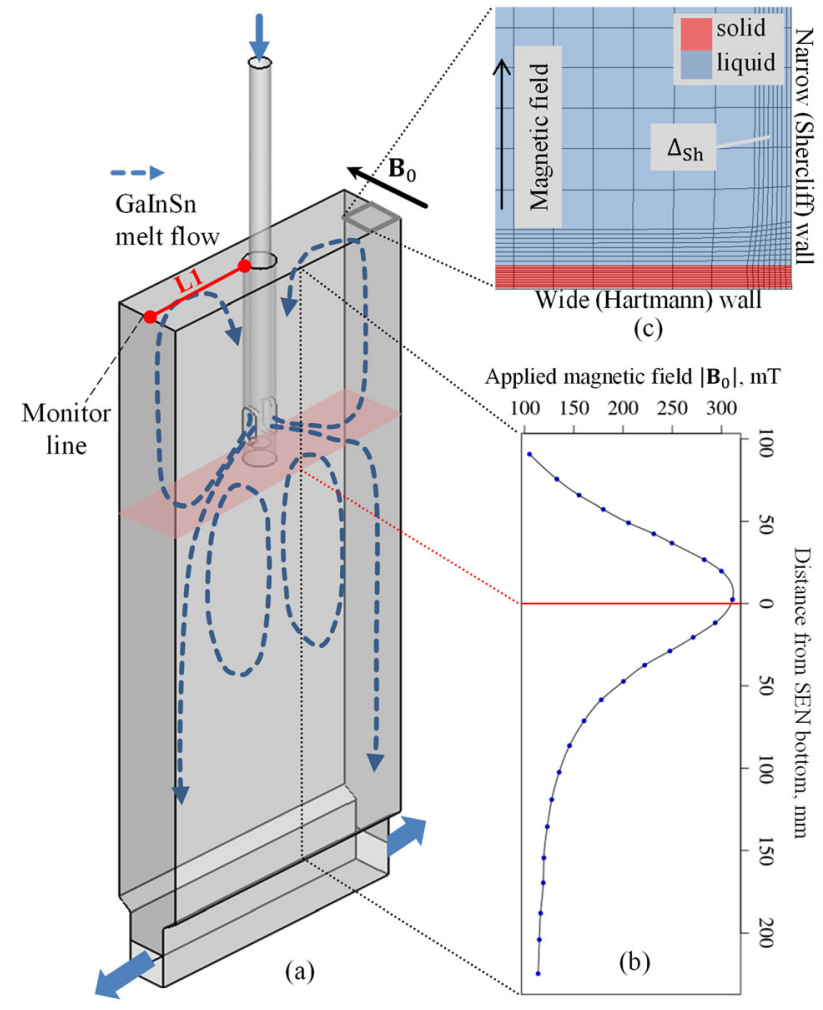

Fig. 1-Overview of the (a) simulated mini-LIMMCAST geometry $\left(140 \times 35 \mathrm{~mm}^{2} \mathrm{CC}\right.$ mold $)$ with the results monitoring line L1, $(b)$ distribution of the applied magnetic field (data taken from Ref. 8), and $(c)$ details of the numerical grid.

The details of the velocity field governed by the EMBr are presented in Figure 4. The upward bending of the jets is typically observed when the magnetic field is applied. ${ }^{[8,10]}$ Recently, it was shown by Schurmann et $a l^{[10]}$ that the EMBr position could have even more impact on the shape of the jets.

Two reverse flow zones are detected above and below the main jet, which are seen both in the midplane section (Figure 4(b)) and at the cross section B-B (Figure 4(c)). This phenomenon can be explained by the fact that the jet becomes elongated along the magnetic field lines between the wide faces, since an essential effect of the magnetic field manifests itself in the distinct reduction of any velocity gradient in the direction of the magnetic field. The continuity of mass requires the melt entrainment from the surroundings of the jet. The latter leads to the formation of a reverse flow at the flattened sides of the jet. ${ }^{[28]}$ The appearance of the reverse flow is confirmed in the experiments. ${ }^{[8]}$

When the wide walls are electrically conductive, the high velocity flow moves along the insulated (narrow) wall. ${ }^{[19]}$ Likewise, a strong upward flow develops along the nonconductive SEN walls under the action of Lorentz force, where an OV is observed. The details for the SEN and narrow wall flow, showing a velocity vector field under applied $312 \mathrm{mT}$ magnetic field, are seen in Figure 4(b). The velocity field of the meniscus and the reverse flow zone above the jet are presented in the cross sections A-A and B-B (Figure 4(c)), respectively. 


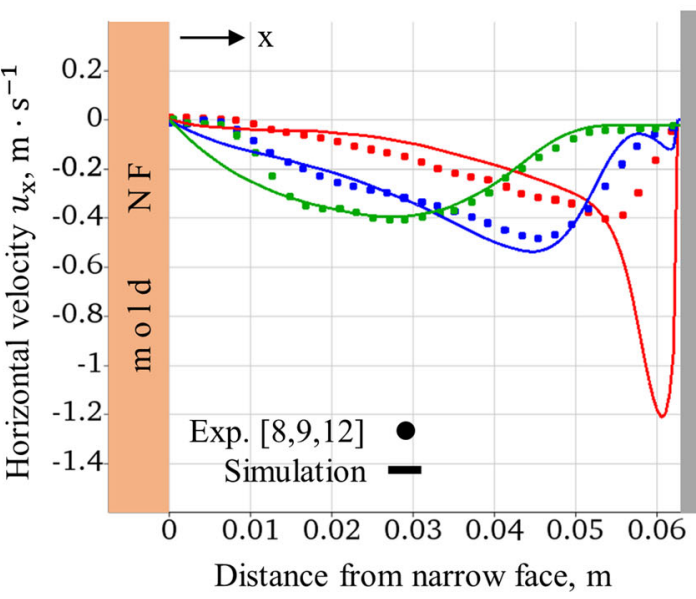

(a)

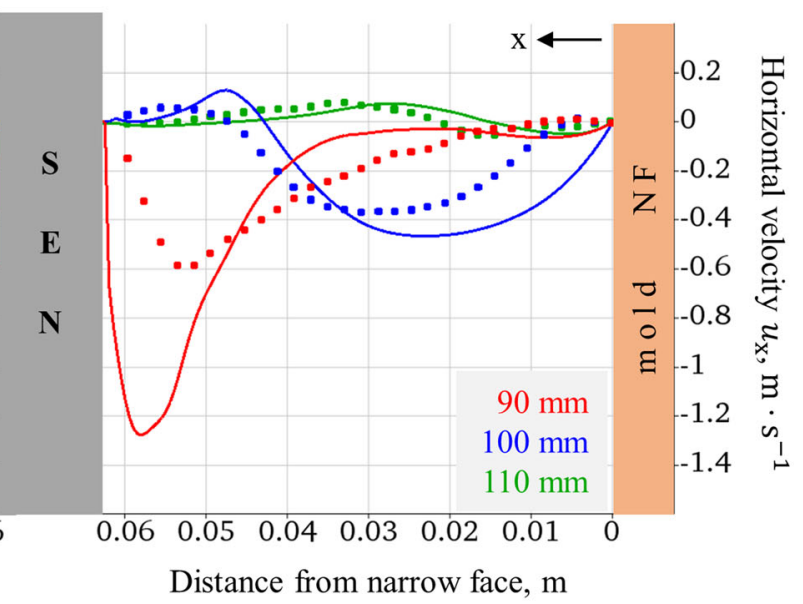

(b)

Fig. 2-Verification of the simulation results based on the mini-LIMMCAST experiment data: ${ }^{[8,9,11,12]}(a)$ flow simulation without EMBr and $(b)$ modeling of the flow under the applied magnetic field $(312 \mathrm{mT})$. The probe lines are located 90,100 , and $110 \mathrm{~mm}$ below the meniscus parallel to L1 in Fig. 1(a).

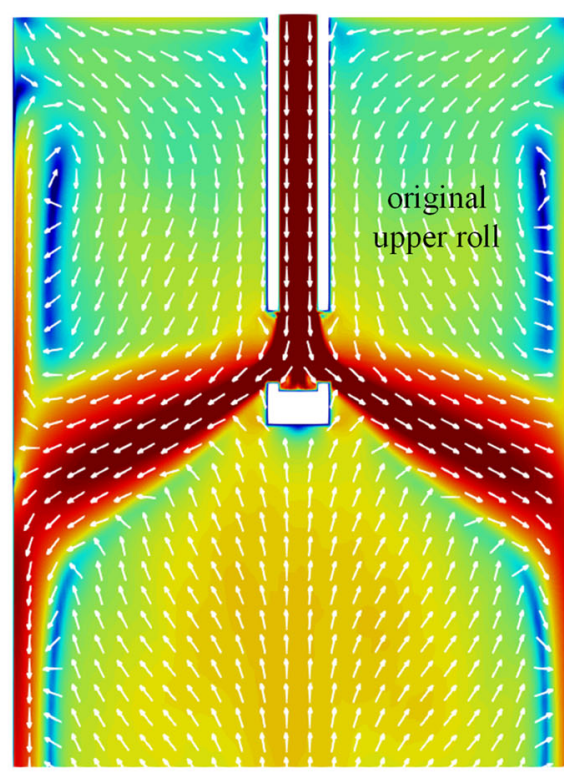

(a)

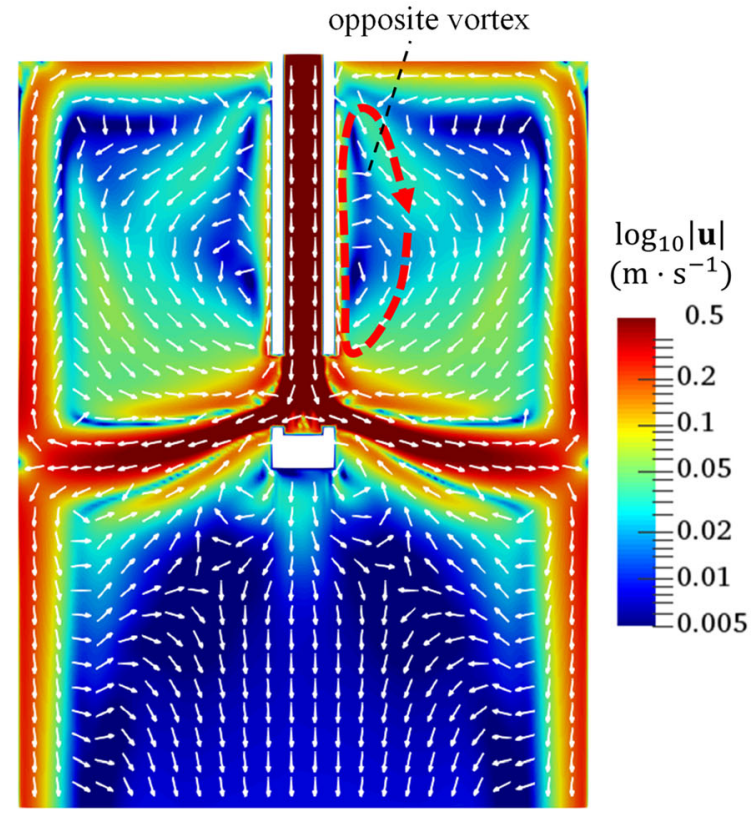

(b)

Fig. 3-Time averaged velocity magnitude distribution in the midplane of the mold cavity for the case $(a)$ without and $(b)$ with active EMBr at a maximum magnetic field value $\left|\mathbf{B}_{0}\right|=312 \mathrm{mT}$.

\section{FORMATION OF THE OPPOSITE MENISCUS VORTEX}

The experimental measurements and numerical results revealed the possibility of an acceleration of the meniscus velocity, when an external DC magnetic field is applied in the zone of the submerged jets. ${ }^{[8,9,11,12,15]}$

In the present parameter study, we focus on the specific case, rigorously discussed in the literature, ${ }^{[8,12]}$ considering the presence of the solid shell by the attached brass plates and using a ruler-type $\mathrm{EMBr}$ applied at the SEN level. We investigate the origin of meniscus acceleration by studying its evolution during variations of the $\mathcal{H a}$ number in 12 precisely selected steps from 0 to 600 . Furthermore, we explore the occurrence of reverse meniscus flow, which is associated with the manifestation of a multiroll flow pattern.

The simulated parameters, which follow a gradual increase of the magnetic field strength and, thus, the characteristic Hartmann number, are summarized in Table I. Case A corresponds to the flow without EMBr, while the other cases reflect the continuous increase of 


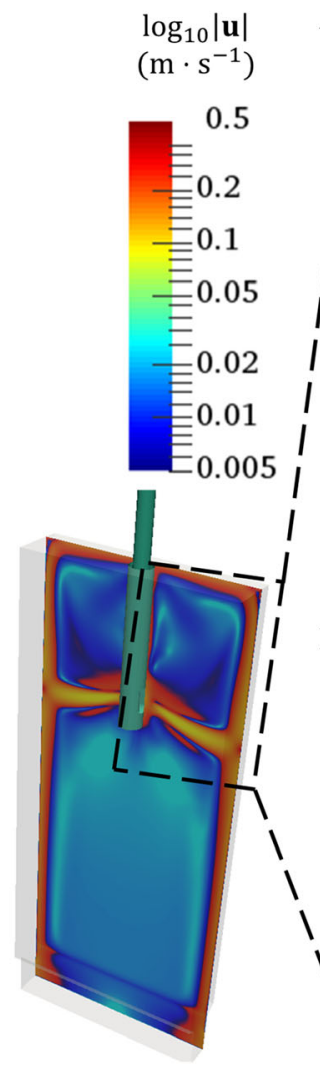

(a)

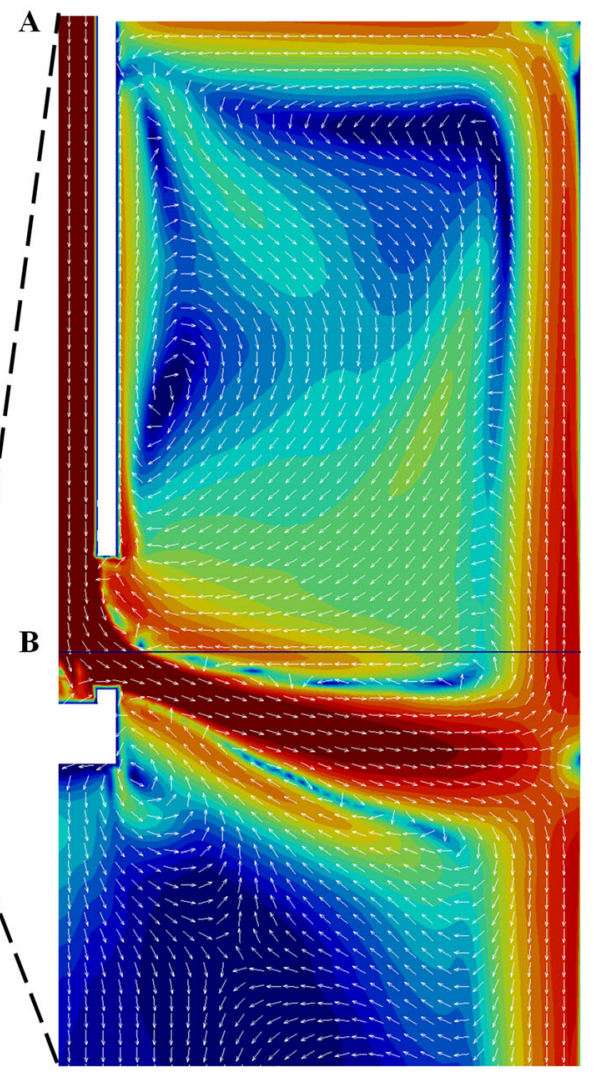

(b)

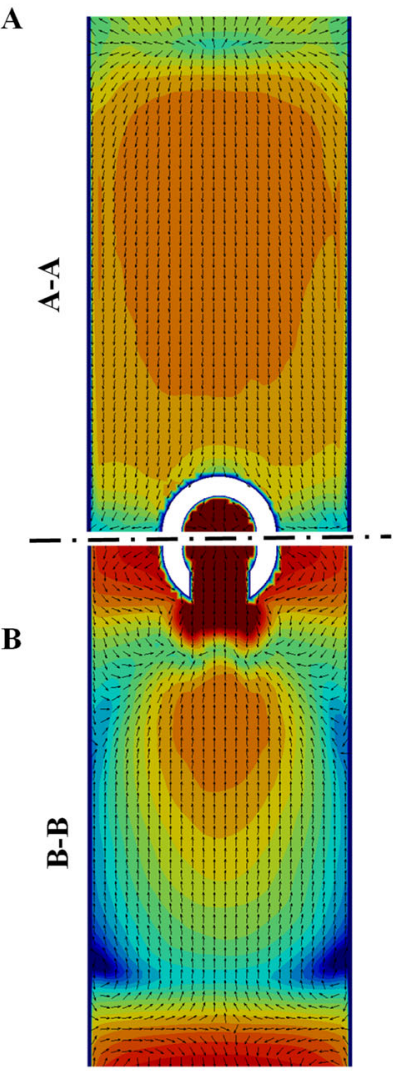

(c)

Fig. 4-Flow direction in the midplane under the influence of the magnetic field $\left|\mathbf{B}_{0}\right|=312 \mathrm{mT}$ : $(a)$ general overview and location of the zoomed areas, $(b)$ jet/SEN wall view, and $(c)$ meniscus velocities (cut A-A) and reverse flow (cut B-B).

the magnetic field, which is expressed by means of the absolute value of the field strength and its relative change compared to the experiment. ${ }^{[12]}$

\section{A. Influence of the Magnetic Field Magnitude: Qualitative Observation}

The first part of the results is shown in Figure 5 as the velocity magnitude distribution in the midplane, where the magnetic field is varied from without $\mathrm{EMBr}$ (case A) up to $221 \mathrm{mT}$ (case E).

Applying a weak field of $39 \mathrm{mT}$ shows almost no changes to the flow pattern (Figure 5(b)). However, when the magnetic field reaches $78 \mathrm{mT}$ (Figure 5(c)), the impact of the $\mathrm{EMBr}$ becomes verifiable: The flow along the narrow wall becomes stronger and the velocities at the meniscus are accelerated as well. This phenomenon becomes dominant at $156 \mathrm{mT}$, where the meniscus velocity reaches its maximum value (Figure 5(d)).

When the EMBr of $221 \mathrm{mT}$ is applied (case E), the meniscus velocity starts to slow. Case $\mathrm{E}$ is the point when the OV is initiated, as marked in Figure 5(e).

Velocity distributions at the meniscus are presented in Figure 6 for cases A through E. It is observed that the meniscus flow is continuously accelerated toward the SEN with growing magnetic field. No significant changes occur in the flow pattern up to a magnetic field value of $156 \mathrm{mT}$. At this point, multiple vortices near the narrow wall (Figures 6(a) through (c)) transform to a single corner vortex apparently aligned with the magnetic field, as shown in Figure 6(d)).

\section{B. Effect on Turbulent Structure}

The action of the applied magnetic field is anisotropic; it does not change the linear momentum of the system, but a transport of vorticity and linear momentum is initiated along the field lines, as pointed out by Davidson. ${ }^{[28]}$ This leads to the formation of quasi-two-dimensional (2-D) flow structures that are not directly affected by Joule dissipation. While three-dimensional (3-D) flows are effectively suppressed, dissipation of the quasi-2-D vortices takes place at sufficiently high field strength in the Hartmann layer in the form of the so-called Hartmann braking. ${ }^{[33,34]}$ To analyze the change of the turbulent flow structure, it is visualized using the so-called Q-criterion, which is estimated based on the velocity gradient as ${ }^{[35]}$

$$
\mathcal{Q}_{\text {crit }}=\frac{1}{2}\left[(\operatorname{tr}(\nabla \mathbf{u}))^{2}-\operatorname{tr}(\nabla \mathbf{u} \bullet \nabla \mathbf{u})\right]
$$


This visualization method is common in the CFD community and defines a vortex structure based on the second invariant of the velocity gradient, representing the local balance between the shear strain rate and vorticity. ${ }^{[36]}$

As shown in Figure 7, there is a clear redistribution of the turbulent structures. Small-scale 3-D vortices are strongly damped, while large-scale 2-D structures become much more prominent. Thus, the flow along the narrow walls toward the meniscus is enhanced when the $\mathrm{EMBr}$ is activated. In fact, part of the turbulent kinetic energy is dissipated in the form of Joule heating, while another part is transferred to the mean flow.

Table I. Simulated Cases Based on the EMBr Magnetic Field Variation

\begin{tabular}{lrrl}
\hline Case & $\left|\mathbf{B}_{0}\right|, \mathrm{mT}$ & $\mathcal{H} a,-$ & \multicolumn{1}{c}{ Comment } \\
\hline A & 0 & 0 & no EMBr case \\
$\mathrm{B}$ & 39 & 52 & - \\
$\mathrm{C}$ & 78 & 104 & - \\
$\mathrm{C}^{*}$ & 117 & 156 & additional study point \\
$\mathrm{D}$ & 156 & 209 & maximum meniscus velocity \\
$\mathrm{E}$ & 221 & 295 & OV initiated \\
$\mathrm{F}^{*}$ & 312 & 417 & experimental setup \\
$\mathrm{G}$ & 349 & 466 & OV reached meniscus \\
$\mathrm{H}$ & 357 & 478 & - OV becomes dominant \\
$\mathrm{I}$ & 366 & 489 & OV bus developed \\
$\mathrm{J}$ & 382 & 511 & meniscus OV fully deint \\
$\mathrm{K}$ & 413 & 552 & additional study point \\
$\mathrm{L}$ & 441 & 590 & OV fully occupies top of CC \\
& & & mold \\
\hline
\end{tabular}

\section{Quantitative Analysis of the Reverse Flow}

For the applied magnetic field of $221 \mathrm{mT}$, it is observed in Figure 8 that the initial OV is developed right above the SEN port exit. It is initiated as a mass compensation for the long reverse flow zones right above the jets, which are marked in Figure 8(a). The existence of the corner vortex at the meniscus in the vicinity of the narrow wall becomes obvious in Figure $8(\mathrm{~b})$. It is parallel to the narrow wall of the mold and rotates opposite to the main meniscus flow.

In a next step, we analyze the evolution of the flow pattern for higher $\mathcal{H a}$ numbers starting from the experimental settings in case $\mathrm{F}$. The results are shown in Figure 9: The OV, which is initiated at a field strength of $221 \mathrm{mT}$ (case E), continuously expands toward the meniscus and reaches the top surface at $349 \mathrm{mT}$ (Figure 9(b)). This is also clearly seen in the meniscus velocity distribution in Figure 10(b).

The newly formed opposite meniscus flow (case I, $366 \mathrm{mT}$ ) progresses from the SEN toward the narrow wall (Figure 9(c)) and merges with the corner vortex in case $\mathbf{J}(382 \mathrm{mT})$, as shown in Figure 9(d). Finally, with the increase in the magnetic field up to $441 \mathrm{mT}$ (case L), the opposite meniscus flow occupies the entire space between the SEN and the narrow wall, as shown in Figure 9(e).

The same phenomenon is presented in Figure 10 from the top view. A clear emergence of the opposite meniscus roll at the top surface, its expansion toward the narrow wall with the increasing strength of the $\mathrm{EMBr}$, and the final merge with the corner roll can be seen in Figures 10(b) through (d).

The detailed development of the submeniscus velocity for the magnetic field in the range between 0 and $349 \mathrm{mT}$ is shown in Figure 11. The time-averaged meniscus velocity immediately starts to grow when the magnetic field is applied. The corresponding range between 0 and $156 \mathrm{mT}$ is displayed with gray colors in Figure 11 and

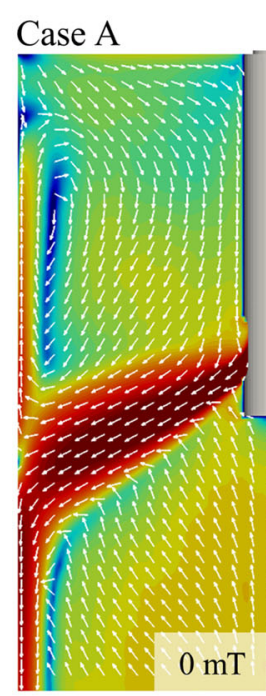

(a)

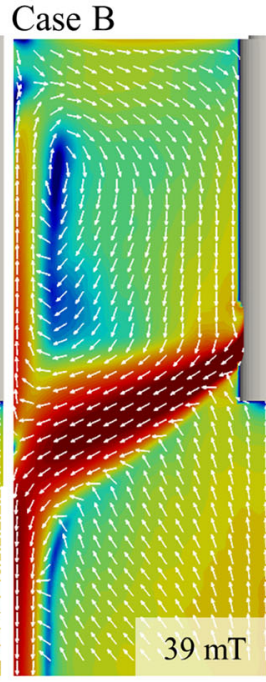

(b)

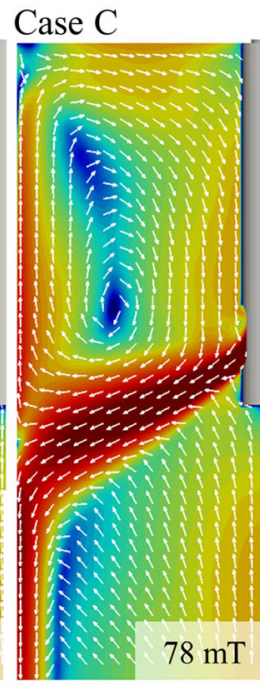

(c)

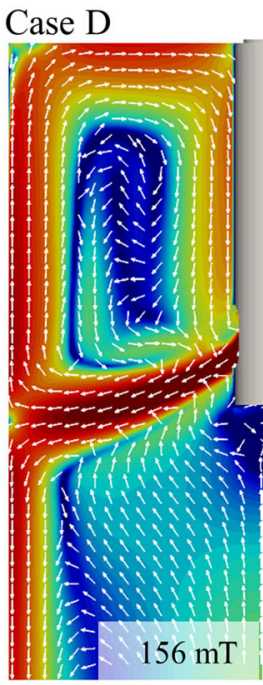

(d)

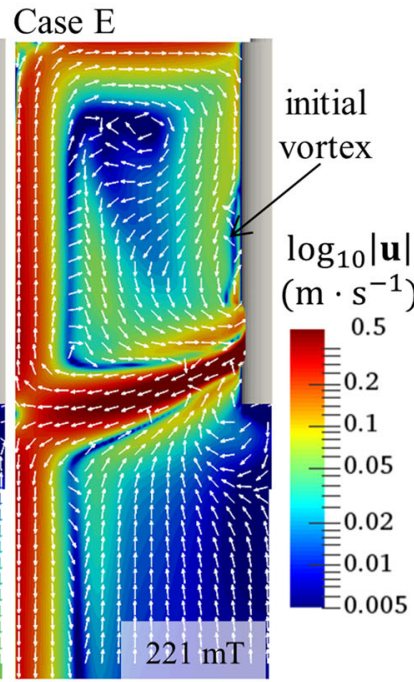

(e)

Fig. 5-Velocity magnitudes and direction in the midplane: (a) no magnetic field and with the applied magnetic field $\left|\mathbf{B}_{0}\right|$ of $(b) 39 \mathrm{mT}$, (c) 78 $\mathrm{mT},(d) 156 \mathrm{mT}$, and $(e) 221 \mathrm{mT}$. 


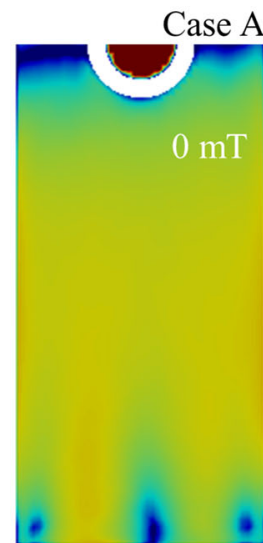

(a)

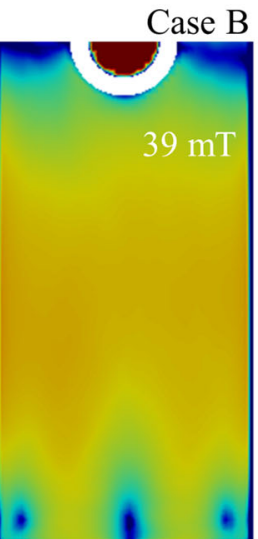

(b)

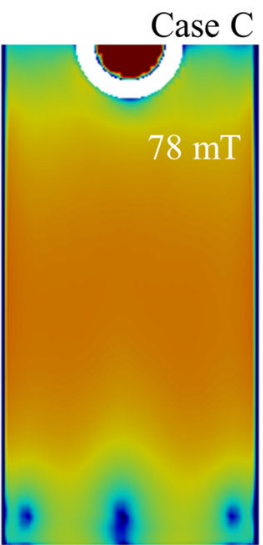

(c)

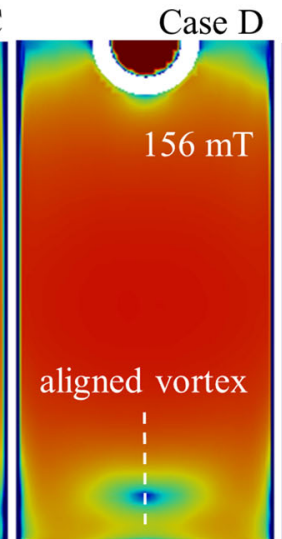

(d)

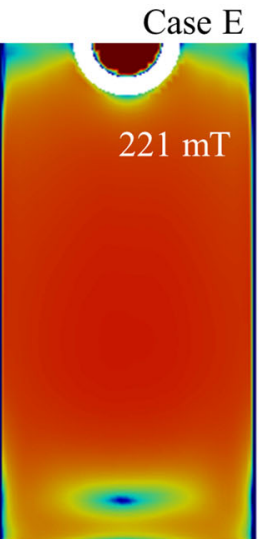

$$
\log _{10}|\mathbf{u}|
$$

$\left(\mathrm{m} \cdot \mathrm{s}^{-1}\right)$

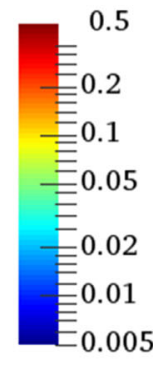

(e)

Fig. 6-Velocity magnitude distribution at the meniscus: (a) no magnetic field and with the applied magnetic field $\left|\mathbf{B}_{0}\right|$ of $(b) 39 \mathrm{mT},(c) 78 \mathrm{mT}$, (d) $156 \mathrm{mT}$, and (e) $221 \mathrm{mT}$.

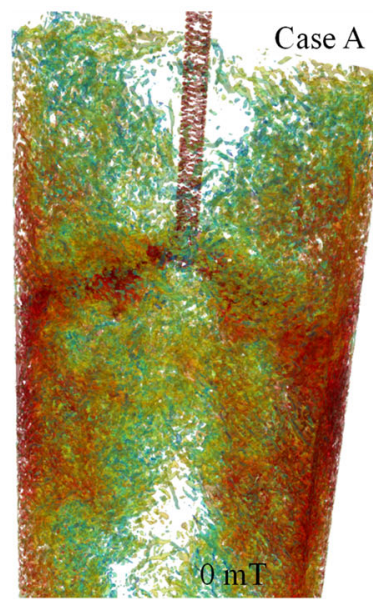

(a)

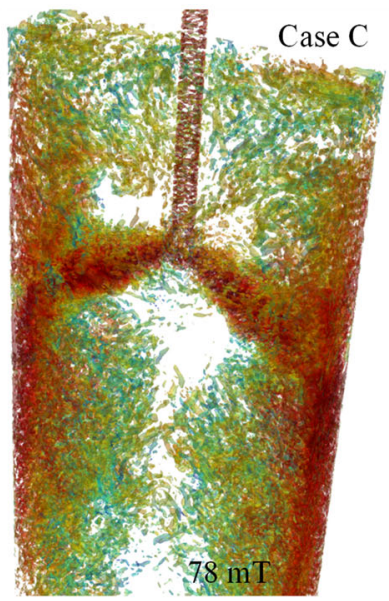

(b)

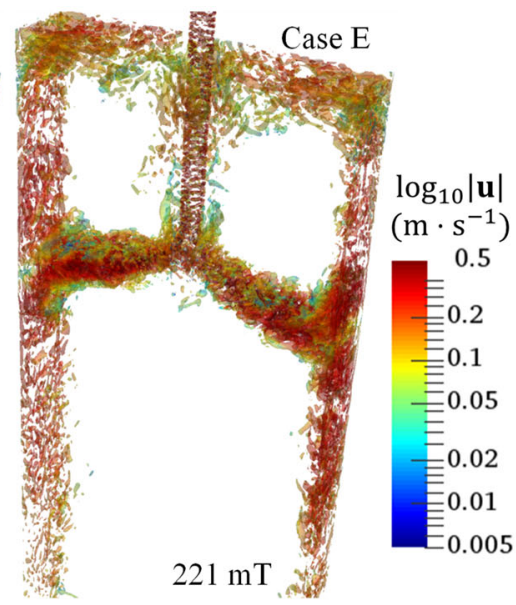

(c)

Fig. 7- Change of the turbulent structures in the mold flow displayed by an isosurface of the Q-criterion $\mathcal{Q}_{\text {crit }}=10$ for $(a)\left|\mathbf{B}_{0}\right|=0 \mathrm{mT}$ (case A), (b) $\left|\mathbf{B}_{0}\right|=78 \mathrm{mT}$ (case C), and (c) $\left|\mathbf{B}_{0}\right|=221 \mathrm{mT}$ (case E; initial OV is formed).

marked with the upward blue arrow. The maximum value of $0.26 \mathrm{~m} / \mathrm{s}$ is reached in the middle section between the narrow wall and the SEN for the magnetic field strength of $156 \mathrm{mT}$.

However, a further increase of the applied magnetic field to $221 \mathrm{mT}$ and continuing to a value of $349 \mathrm{mT}$ causes a reduction of the meniscus flow again; it is marked with the downward arrow in Figure 11. Furthermore, the development of a corner vortex at the narrow wall can be observed. Its intensity appears to behave in the same way as the velocity value of the dominant submeniscus flow.

A qualitative change of the meniscus flow pattern occurs in case $\mathrm{G}$ (marked with a dash line) for $\left|\mathbf{B}_{0}\right|=$ 349 mT: A top part of the OV occurs at the meniscus close to the SEN wall (also Figures 9 through 10(b)) and the transition to the multiroll flow regime starts.
Further details are given in Figure 12, containing the horizontal velocity component profiles at the meniscus for the $\mathrm{EMBr}$ in the range between 312 and $441 \mathrm{mT}$. It should be mentioned that an additional case $\mathrm{K}(\mathrm{EMBr}$ $413 \mathrm{mT}$ ) is used to show the asymptotic behavior of the opposite meniscus flow development. It is shown with a thin blue dash line in Figure 12 and lies very close to the line of $441 \mathrm{mT}$ (case L). Since a very negligible difference in the flow pattern with the stronger $\mathrm{EMBr}$ (case L) is detected, it is not necessary to go for the magnetic field values above $441 \mathrm{mT}$.

With the fully developed multiroll, the flow direction in the upper region of the mold is inversed in comparison with the initial double roll pattern. Here, in case L, the highest speed is around $0.15 \mathrm{~m} / \mathrm{s}$ near the SEN and continuously decreases to $0.07 \mathrm{~m} / \mathrm{s}$ close to the narrow 


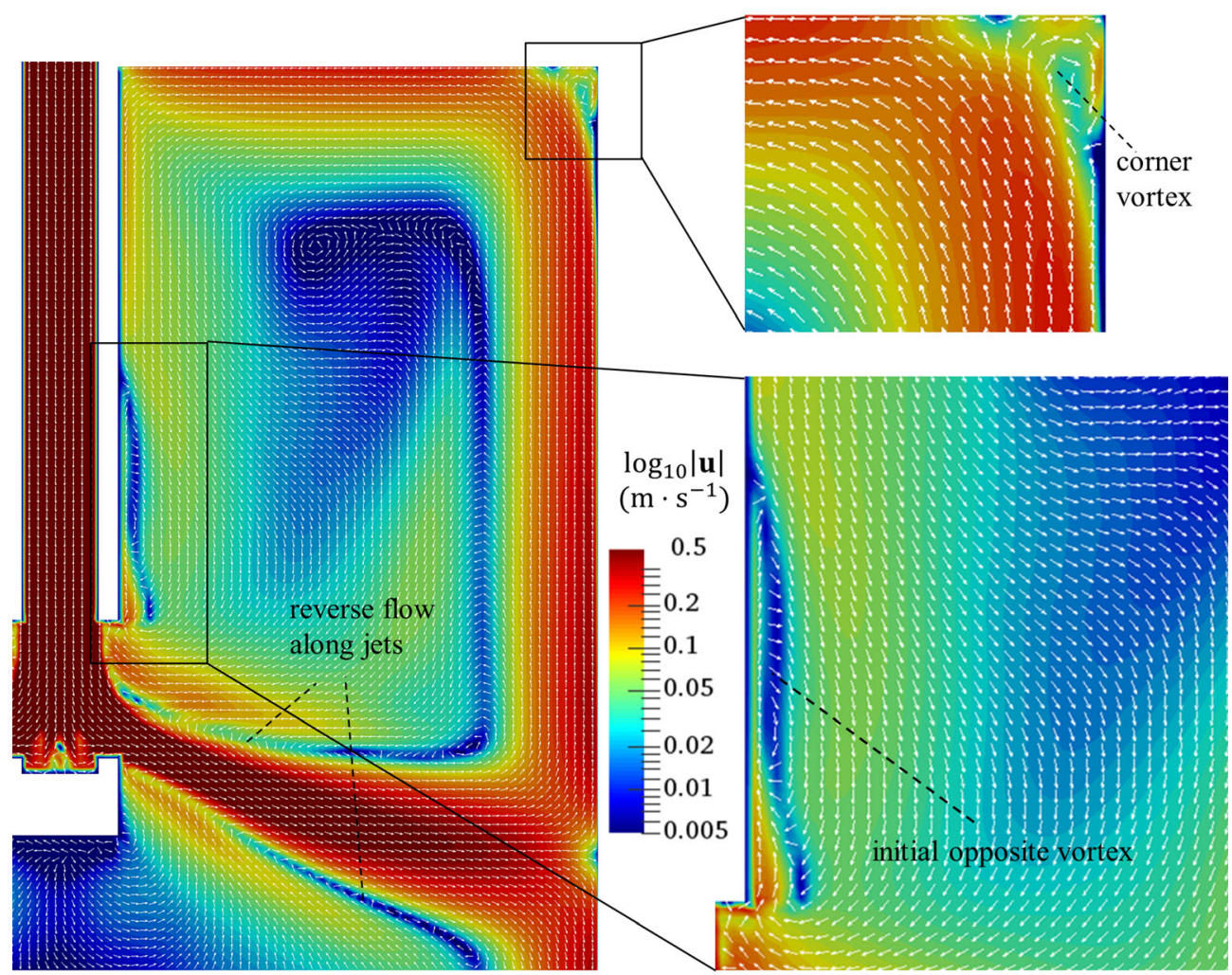

(a)

(b)

Fig. 8-Flow pattern for $\left|\mathbf{B}_{0}\right|=221 \mathrm{mT}$ : (a) general overview and (b) details of the initial OV formation near the SEN and the corner vortex near the meniscus.

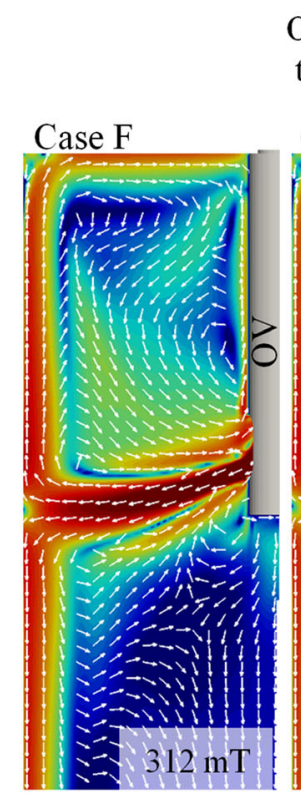

(a)
OV reaches top surface

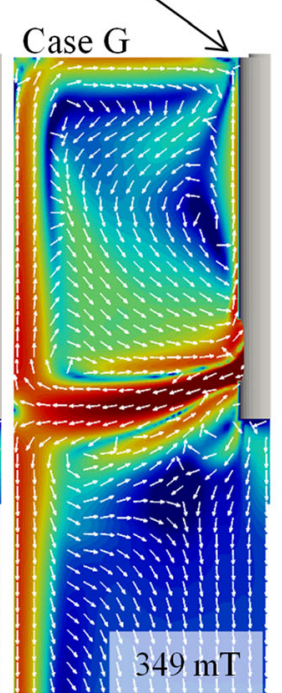

(b)

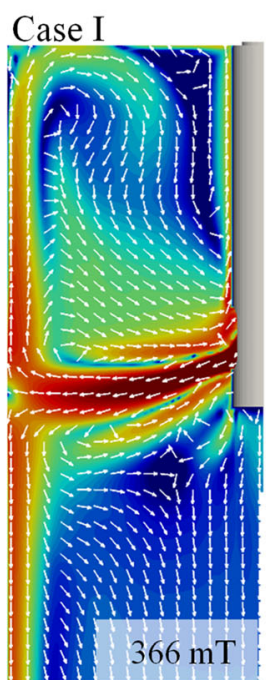

(c)

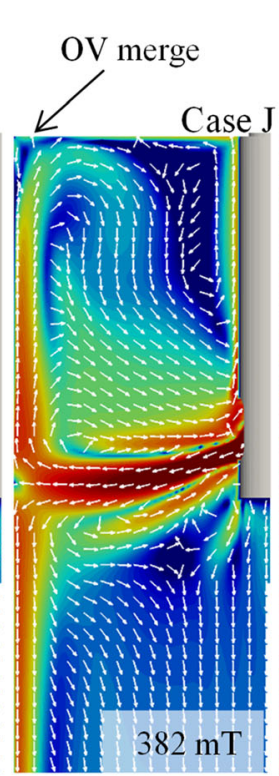

(d)

\section{OV fully \\ developed}

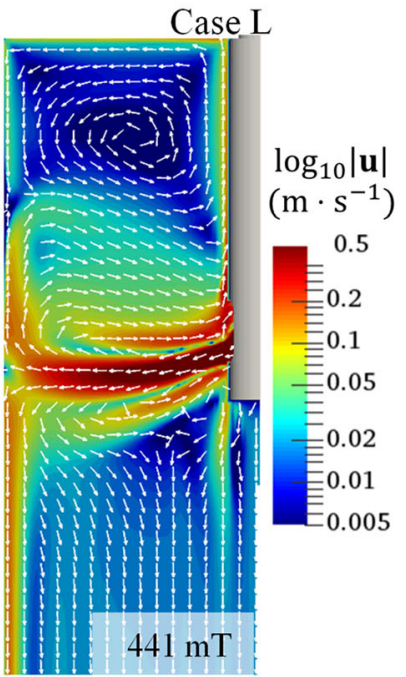

(e)

Fig. 9-Velocity magnitude distribution and flow direction in the midplane during the OV growth and the transition between the double and the multiroll (reverse flow) regimes: (a) $\left|\mathbf{B}_{0}\right|=312 \mathrm{mT}$, (b) $\left|\mathbf{B}_{0}\right|=349 \mathrm{mT},(c)\left|\mathbf{B}_{0}\right|=366 \mathrm{mT}$, (d) $\left|\mathbf{B}_{0}\right|=382 \mathrm{mT}$, and (e) $\left|\mathbf{B}_{0}\right|=441 \mathrm{mT}$. 


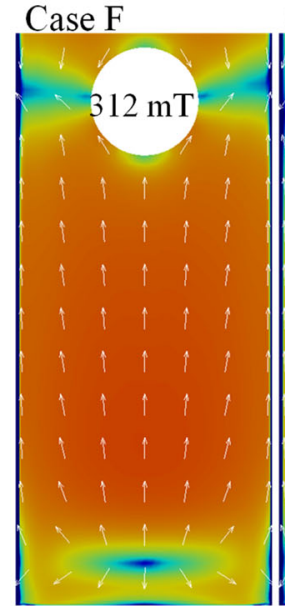

(a)
Case $\mathrm{G}$

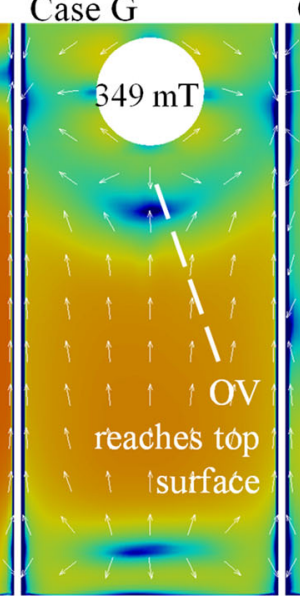

(b)

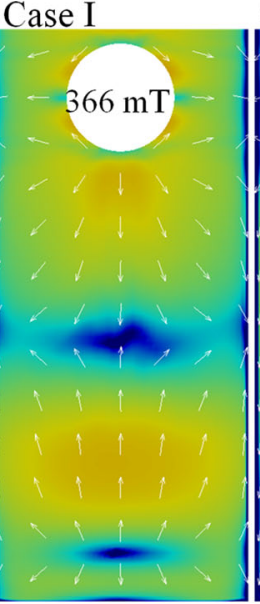

(c)

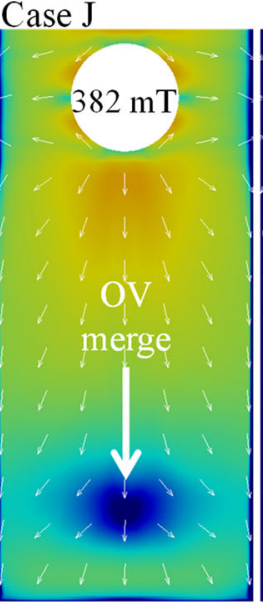

(d)
Case L

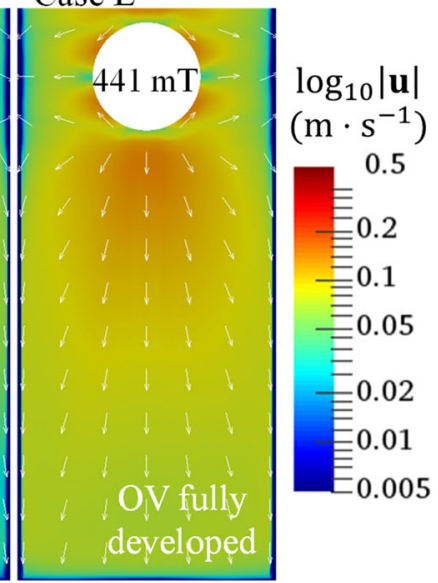

(e)

Fig. 10-Flow direction and velocity magnitudes at the meniscus during the OV growth and the transition between the double and the multiroll (reverse flow) regimes: (a) $\left|\mathbf{B}_{0}\right|=312 \mathrm{mT}$, (b) $\left|\mathbf{B}_{0}\right|=349 \mathrm{mT}$, (c) $\left|\mathbf{B}_{0}\right|=366 \mathrm{mT}$, (d) $\left|\mathbf{B}_{0}\right|=382 \mathrm{mT}$, and (e) $\left|\mathbf{B}_{0}\right|=441 \mathrm{mT}$.

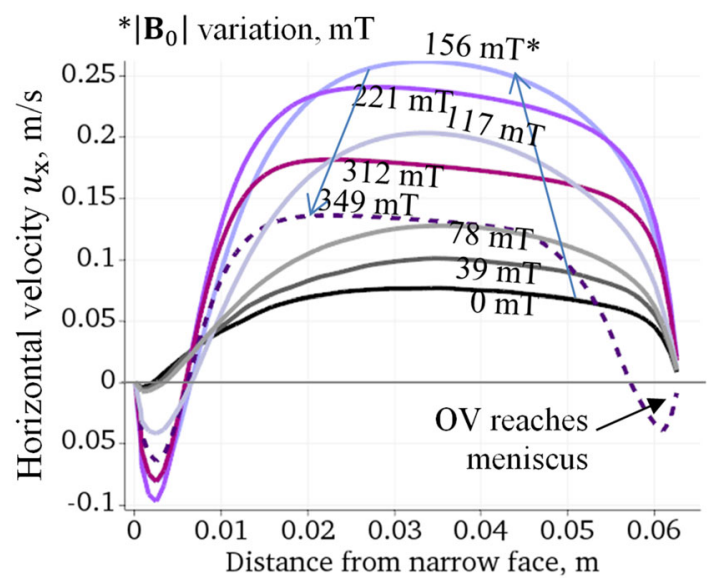

Fig. 11-Meniscus time-averaged horizontal velocity $u_{x}$ distribution along the monitoring line $\mathbf{L} 1$ in Fig. 1 for cases A through $G$ (0 to $349 \mathrm{mT})$.

wall. For the typical converging meniscus, the highest speed $(\sim 0.18 \mathrm{~m} / \mathrm{s})$ is found to occur closer to the NFs and decrease toward the SEN.

Figure 13 focuses on a section of the velocity profiles near the narrow wall, showing in detail how the corner vortex there develops under the influence of the magnetic field. In the real casting process, such corner vortices also occur due to shell withdrawal. Vakhrushev et $a l .{ }^{[4]}$ showed that both types of corner vortices can combine and reinforce each other, possibly leading to an enhanced entrainment of liquid slag into the mold/shell gap.

Figure 13 also makes it obvious that the higher the magnetic field, the further the corner vortex extends into the mold. A maximum velocity of about $0.08 \mathrm{~m} / \mathrm{s}$ appears at a magnetic flux density of $312 \mathrm{mT}$. Further increase of $\mathbf{B}_{0}$ up to $382 \mathrm{mT}$ reduces the velocity to about $0.04 \mathrm{~m} / \mathrm{s}$ before the opposite roll merges with the corner vortex and covers the entire liquid surface (case L).

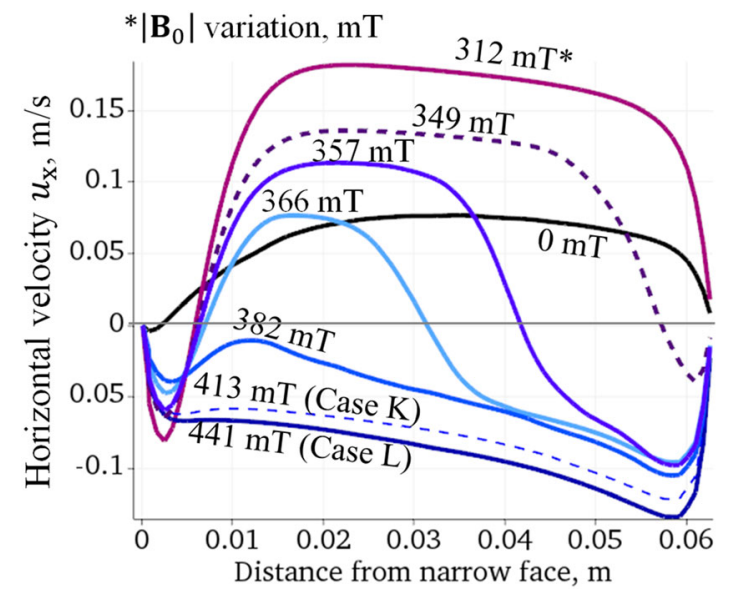

Fig. 12-Meniscus time-averaged horizontal velocity $u_{x}$ distribution velocity along the monitoring line $\mathbf{L} 1$ in Fig. 1 for cases $\mathrm{F}$ through $\mathrm{L}$ (312 to $441 \mathrm{mT}$ )

\section{Action of the Lorentz Force}

To assess the action of the Lorentz force, a special function is defined as follows:

$$
\mathbf{L}\left(\mathbf{F}_{L}, \mathbf{u}\right)=\frac{\mathbf{F}_{L} \bullet \mathbf{u}}{\left|\mathbf{F}_{L}\right| \bullet|\mathbf{u}|}
$$

which represents a normalized dot product of the Lorentz force and the melt velocity in the range between 1 and +1 . The negative $\mathbf{L}\left(\mathbf{F}_{L}, \mathbf{u}\right)$ corresponds to damping, while the positive value means that, locally, the Lorentz force accelerates the flow.

According to Eq. [5] for the magnetic field, applied in the normal direction to the mold's wide face, the Lorentz force will act in the vertical plane only. The distribution of the Lorentz force function $\mathbf{L}\left(\mathbf{F}_{L}, \mathbf{u}\right)$ in the vertical center plane is investigated in Figure 14 together with the magnitude of the magnetic force and the induced current density to distinguish wherever its 
action is important or not. A colored stripe on the very left of Figure 14 indicates the EMBr location and intensity.

The regime where the $\mathrm{OV}$ is initialized (case $\mathrm{E}$, $221 \mathrm{mT}$ ) is analyzed in Figure 14(a): The Lorentz force brakes the flow in jets, along the narrow walls, inside the upper rolls as well as below the SEN. However, as shown by Schurmann et al. ${ }^{[10]}$ the Lorentz force does not decelerate the jets; it flattens the exit angle of the flow, transforming it from "banana" shape to almost parallel or even to " $\mathrm{S}$ " shape (Figure 14, top row). The $\mathrm{EMBr}$ force accelerates the flow at $221 \mathrm{mT}$ in the lower part of the mold; however, the magnitude is weak, and the effect is negligible.

With the initiation of the $\mathrm{OV}$, a braking zone appears near the SEN wall right above the port outlet. Despite the fact that it goes outside the EMBr effective range, the Lorentz force magnitudes are not negligible close to the top surface since the induced currents are concentrated at the upper part of the mold and continuous flow structure reorganization happens. The braking zone develops as the OV grows toward the meniscus. Simultaneously, the flow is accelerated in the upper roll. Thereby, the OV grows to satisfy the mass conservation. For cases $\mathrm{H}$ and $\mathbf{J}$ (Figures 14(b) through (c)), the pronounced acceleration zones (red) are detected above and below the main jets in the reverse flow region. The detailed formation of these recirculation zones is previously discussed and is presented in Figure 9.

For an applied magnetic field of $441 \mathrm{mT}$ (Figure 14(d)), the OV is fully developed along the top surface (dashed line with an arrow). It fully occupies the upper part of the mold and is under acceleration below the meniscus. However, the main acceleration zone is now in the upper roll (solid line with an arrow). In comparison to the standard double-roll flow, the upper roll is now pushed deep under the meniscus surface due to the action of the Lorentz force.

Right below the SEN, the Lorentz force dominantly acts as a braking mechanism of the flow. Its value is significant due to the high magnetic field values in the effective EMBr zone, leading to the plug-type flow at the lower part of the domain resulting in a uniform downward motion of the melt.

It should be emphasized that the action of the Lorentz force cannot be reduced by its damping effects. As seen from the results in Figure 14, the MHD force action is very complex and its topology significantly changes under the growing magnetic field.

\section{E. Transition from Double to Multiroll Based on the Hartmann Number}

To summarize the studies for the EMBr positioning at the SEN bottom (92 $\mathrm{mm}$ below the top surface), the dependency of the maximum meniscus velocity based on the Hartmann number is shown in Figure 15. It can be detected that the meniscus accelerates starting from the case without $\mathrm{EMBr}$ to the case where the Hartmann number reaches 200. Then a velocity drop is observed since internal vortices develop inside the bulk region withdrawing kinetic energy from the meniscus. As marked in Figure 15, for the case I, the upper roll and the opposite meniscus become equally strong. That finally leads to the fully developed opposite meniscus vortex at $\mathcal{H} a \approx 510$. The flow at the top surface changes its direction toward the narrow walls of the mold cavity.

The double roll regime, despite momentum redistribution, is kept in the range up to $\mathcal{H} a \approx 300$. Further, with the growth of the magnetic field, the OV is formed. Afterward, the competition starts between the Lorentz force action and the momentum conservation in the liquid flow. The sizes of the OV and the upper roll are comparable in this regime. However, with Hartmann numbers in the range between 450 and 550, the flow pattern totally changes. The bottom part represents plug flow, the jets are surrounded by recirculation zones above and below them, and the upper part of the mold is totally occupied by the reverse flow.

\section{F. Influence of the Shell Location and Withdrawal}

In the real casting process, the solidifying shell is continuously growing against a water-cooled copper mold; additionally, the CC slab is being withdrawn with a corresponding casting speed. To reveal the importance of the shell distribution and its withdrawal on the flow pattern under the EMBr, two additional studies were performed (Figure 16). The initial setup in this article with the solid shell at the wide walls is used as a reference (Figure 16(a)). Next, an additional brass plate attachment to the narrow walls is considered (Figure 16(b), case (i)). Finally, the movement of the solid shell is included ((Figure 16(b), case (ii)).

The alternation of the horizontal meniscus velocity $u_{x}$ is shown in Figure 16(c). When the brass plates are attached to the wide and narrow walls, enhanced braking of the meniscus is detected, displayed by a shift between the black and blue lines in Figure 16(c). When a pulling velocity of the shell is defined, the meniscus flow slightly accelerates to compensate for additional downward motion in the vicinity of the mold walls (blue and

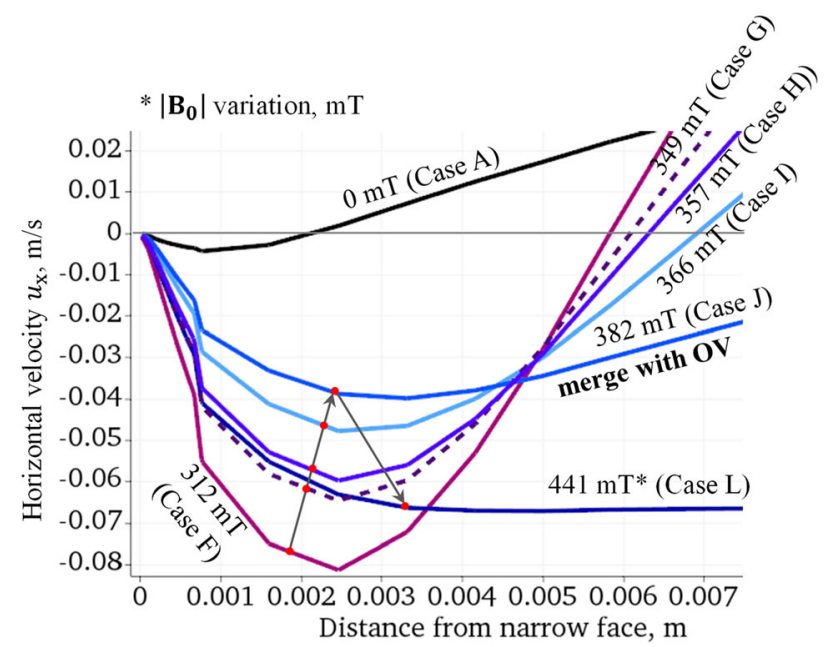

Fig. 13-Progress of the corner vortex for cases $\mathrm{F}$ through $\mathrm{L}$ : the suppression of the corner vortex (upward arrow) until it merges with the OV (downward arrow). 

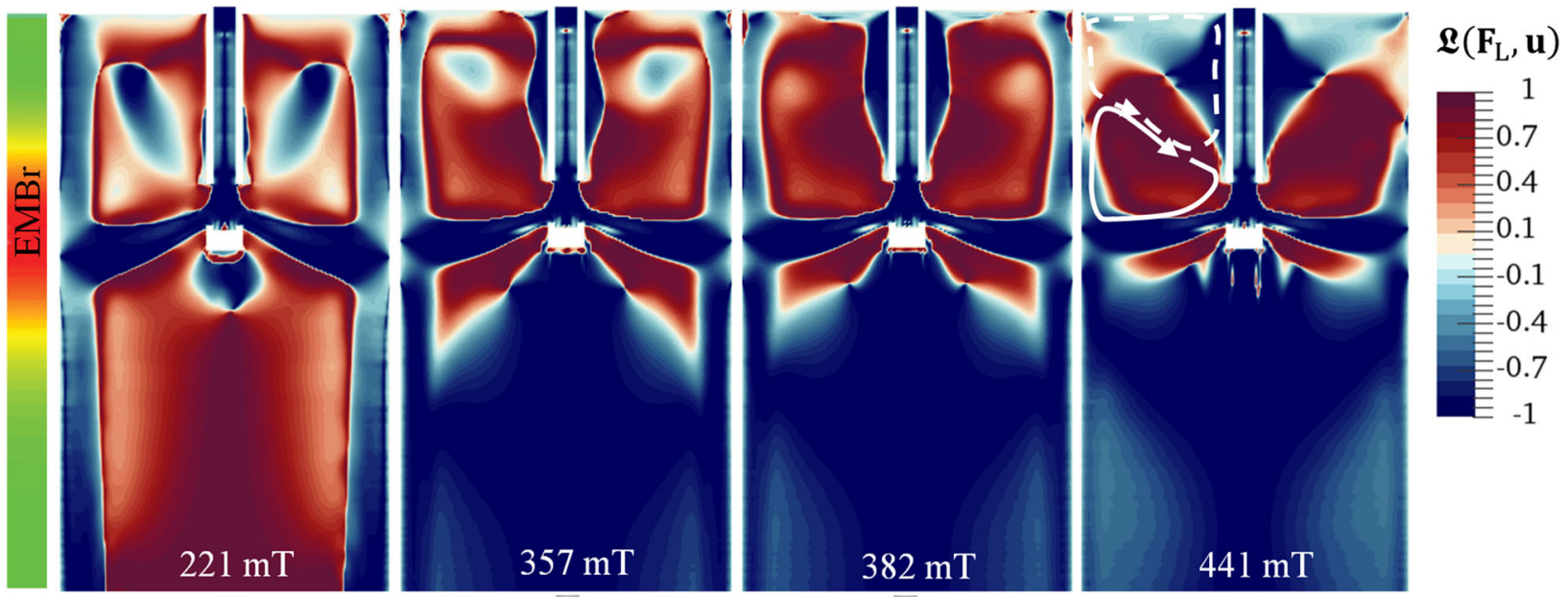

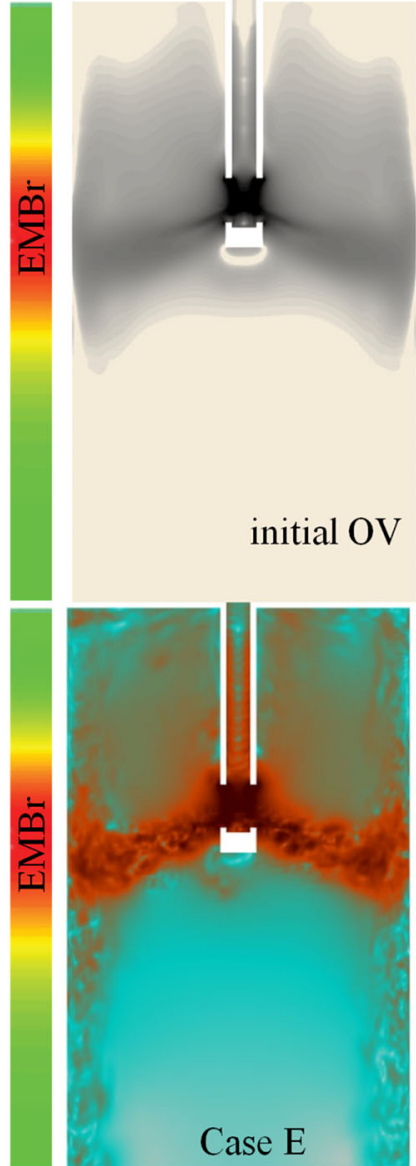

(a)
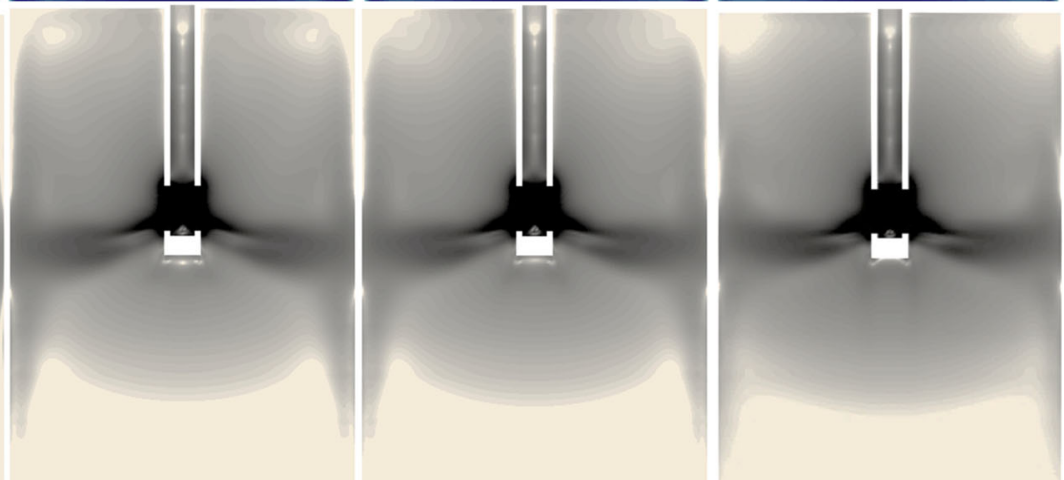

OV fully developed

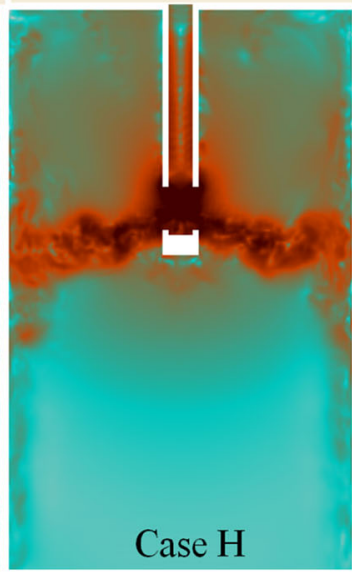

(b)

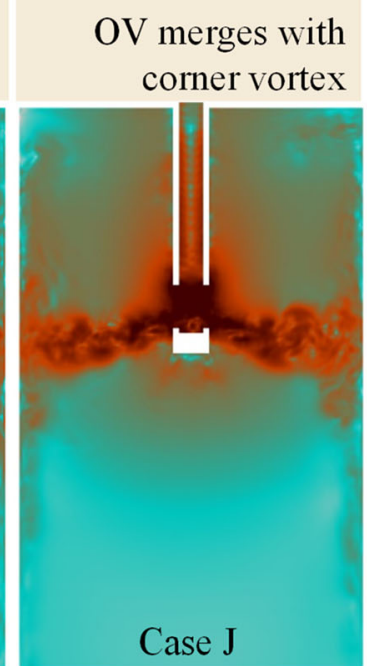

(c)

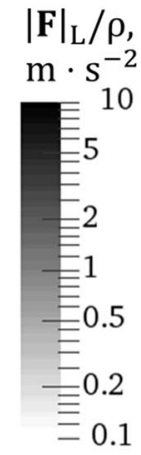

$\log _{10}|\mathbf{j}|$

$\left(\mathrm{A} \cdot \mathrm{m}^{-2}\right)$

300000
-100000

$-50000$

$-20000$

$-10000$

$-5000$

$-2000$

$-1000$

300

Fig. 14 -Distribution of the time-averaged Lorentz force function $\mathbf{L}\left(\mathbf{F}_{L}, \mathbf{u}\right)$ (upper row) and the magnitude of the Lorentz force density (acceleration, midrow) during the opposite meniscus vortex $(\mathrm{OV})$ development; instantaneous distribution of the induced current density (bottom row): (a) $\left|\mathbf{B}_{0}\right|=221 \mathrm{mT}$, (b) $\left|\mathbf{B}_{0}\right|=357 \mathrm{mT},(c)\left|\mathbf{B}_{0}\right|=382 \mathrm{mT}$, and (d) $\left|\mathbf{B}_{0}\right|=441 \mathrm{mT}$.

red lines in Figure 16(c)). However, the difference is not dramatic and the flow pattern is conserved. On the other hand, for the faster casting speeds, one would expect significant changes; therefore, a simplification of the experimental and numerical models should be carefully selected.
We encourage the readers to observe the supplemental materials, including the animation videos "Video S1.avi", "Video S2.avi", "Video S3.avi," and "Video S4.avi," where the most representative results of the flow alternation and the formation of the multiroll pattern under the applied magnetic field are shown. 


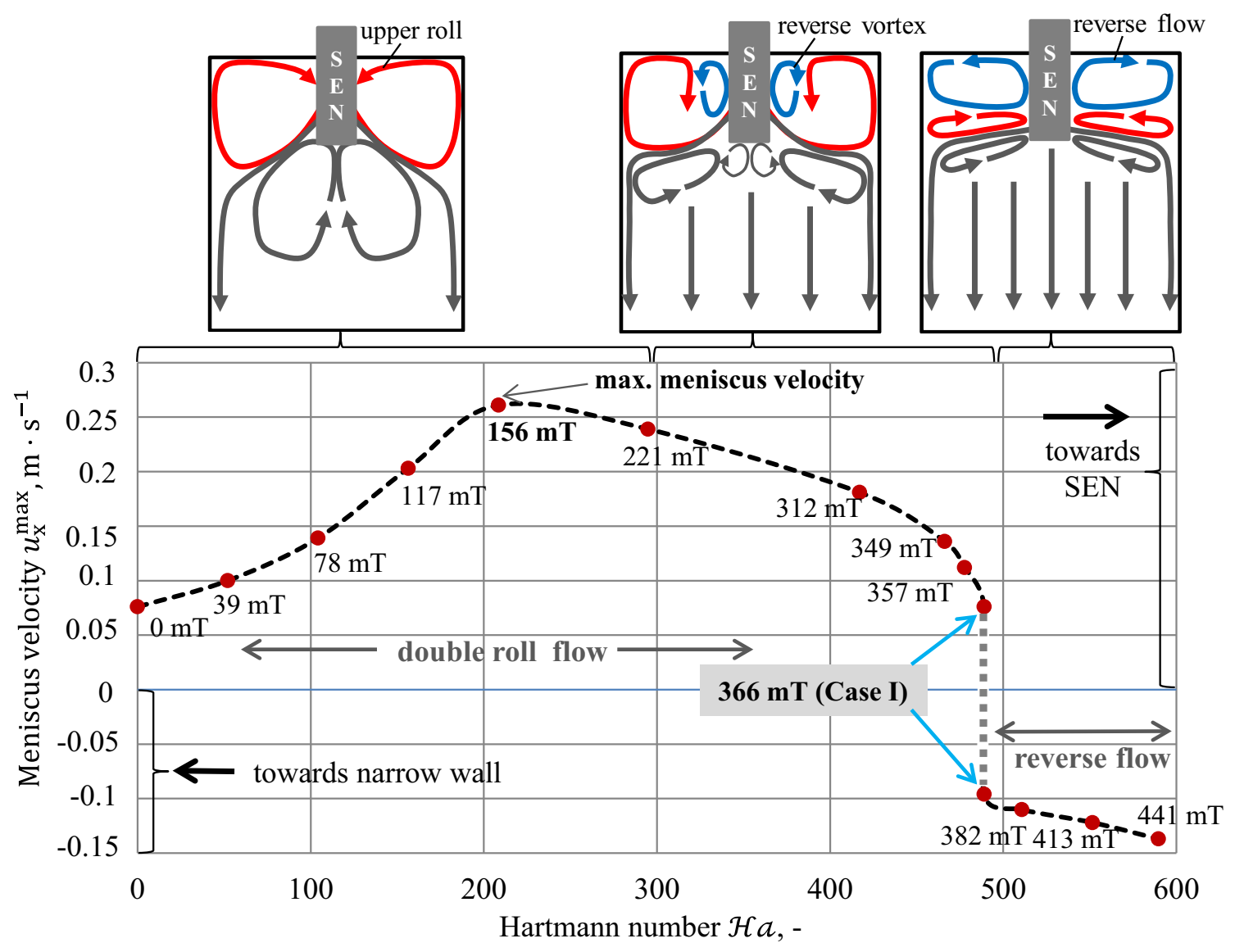

Fig. 15-Mold flow structure based on the Hartmann number $\mathcal{H} a \approx 300$ : Evolution of the maximum (by magnitude) horizontal meniscus velocity $u_{x}^{\max }$ is shown with black dashed line, the peek magnetic field value is marked next to each simulated case, and the overall flow regime is schematically defined at the top of the figure.

\section{CONCLUSIONS}

During this investigation, it was revealed that the meniscus flow undergoes different regimes during the increase of the applied magnetic field. First, it is accelerated since the turbulent structures are damped and all linear momentum from the freshly fed melt is transformed into the upward and downward flow. Thereby, the upper rolls are supported with stronger momentum. Accordingly, at this stage, the meniscus is accelerated as well.

Meanwhile, a reverse flow develops at the SEN port corner. It was generally assumed that this OV is caused by the surrounding melt entrainment due to the mass conservation. The present investigation shows that this reverse flow is of a pure MHD nature. It comes from the induced electric current closure. In convection-dominant areas, it acts in the form of braking. However, in other regions, this current accelerates the flow, giving rise to the formation of a reverse flow adjacent to the jet flow. With the growing magnetic field, the reverse flow reaches the top surface and finally occupies the entire mold width from the SEN to the narrow wall. Consequently, with this OV development, the top surface velocity is initially decreased since the kinetic energy is consumed for the new flow structure formation. However, at some critical intensity of the magnetic field, the meniscus starts to accelerate again. The industrial effect of the fully developed opposite meniscus flow is not fully understood. It can have positive or negative consequences on the product quality. Providing more superheat to the stagnation zones at the clearance between the SEN and the wide walls is, for example, favorable for continuous casting. The consequent enhancement of the slag entrapment possibility is, nevertheless, undesirable.

As revealed in the performed studies, with the variation of the EMBr magnetic field, all significant changes happen in the liquid bulk long before they are observable at the meniscus. This fact should be seriously considered, since most of the measurement techniques for the real casting act close to the slag band level and can give misleading indications.

\section{ACKNOWLEDGMENTS}

The authors acknowledge the financial support by the Austrian Federal Ministry of Economy, Family and Youth and the National Foundation for Research, Technology and Development within the framework of the Christian Doppler Laboratory for Metallurgical Applications of Magnetohydrodynamics. 


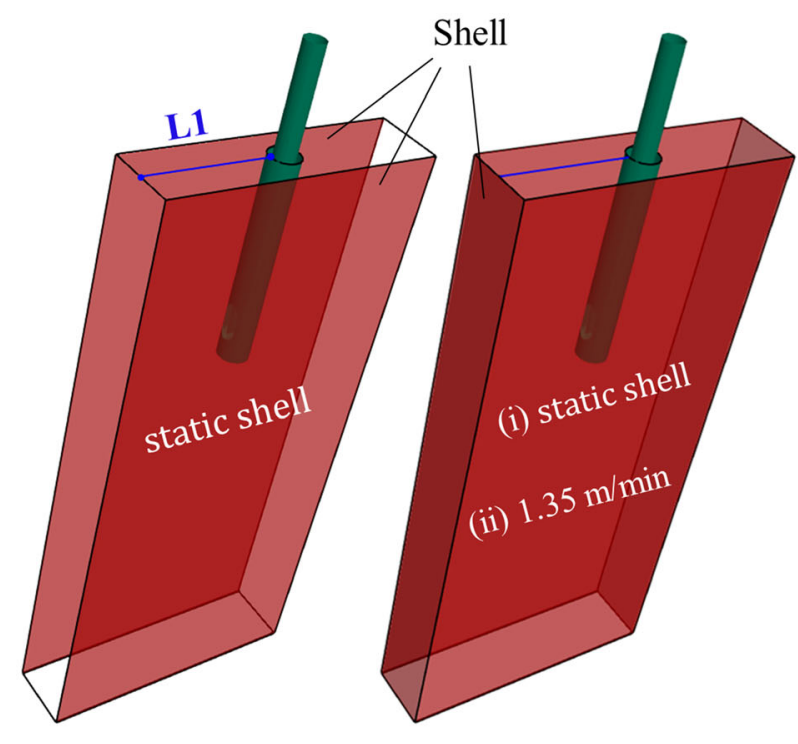

(a)

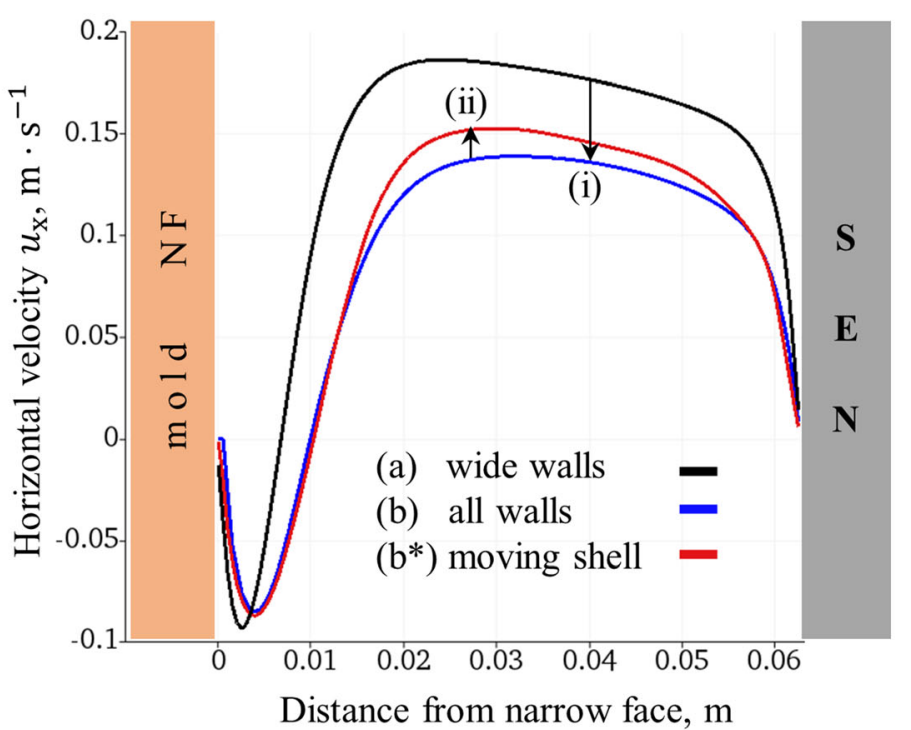

(c)

Fig. 16 - Comparison of the simulated meniscus velocity $u_{x}$ for the solid shell located $(a)$ at wide walls and (b) at wide and narrow walls: (i) static shell and (ii) moving shell with the casting speed $u_{\text {pull }}=1.35 \mathrm{~m} / \mathrm{min}$. (c) $u_{x}$ distribution along the L1 line in Fig. 1(a).

\section{FUNDING}

Open access funding provided by Montanuniversität Leoben.

Open Access This article is licensed under a Creative Commons Attribution 4.0 International License, which permits use, sharing, adaptation, distribution and reproduction in any medium or format, as long as you give appropriate credit to the original author(s) and the source, provide a link to the Creative Commons licence, and indicate if changes were made. The images or other third party material in this article are included in the article's Creative Commons licence, unless indicated otherwise in a credit line to the material. If material is not included in the article's Creative Commons licence and your intended use is not permitted by statutory regulation or exceeds the permitted use, you will need to obtain permission directly from the copyright holder. To view a copy of this licence, visit http://creativecommons. org/licenses/by/4.0/.

\section{SUPPLEMENTARY INFORMATION}

The online version contains supplementary material available at (https://doi.org/10.1007/s11663-021-02247-x).

\section{REFERENCES}

1. S. Cuevas, B.F. Picologlou, J.S. Walker, and G. Talmage: Int. J. Eng. Sci., 1997, vol. 35 (5), pp. 485-503.
2. S. Cuevas, B.F. Picologlou, J.S. Walker, G. Talmage, and T.Q. Hua: Int. J. Eng. Sci., 1997, vol. 35 (5), pp. 505-14.

3. M. Wu, A. Vakhrushev, G. Nunner, C. Pfeiler, A. Kharicha, and A. Ludwig: Open Transp. Phenom. J., 2010, vol. 2 (1), pp. 16-23.

4. A. Vakhrushev, M. Wu, A. Ludwig, Y. Tang, G. Hackl, and G. Nitzl: Metall. Mater. Trans. B, 2014, vol. 45B, pp. 1024-37.

5. M. Wu, A. Vakhrushev, A. Ludwig, and A. Kharicha: IOP Conf. Ser., 2016, vol. 117, art. no. 012045https://doi.org/10.1088/1757$899 X / 117 / 1 / 012045$.

6. Z. Tigrine, F. Mokhtari, A. Bouabdallah, A. Merah, and A. Kharicha: ASME J. Fluids Eng., 2017, vol. 139 (6), art. no. 061201https://doi.org/10.1115/1.4035636.

7. A. Kharicha, A. Ludwig, and M. Wu: Mater. Sci. Eng. A, 2005, vols. 413-414, pp. 129-34.

8. K. Timmel, S. Eckert, and G. Gerbeth: Metall. Mater. Trans. B, 2011, vol. 42B, pp. 68-80.

9. R. Chaudhary, C. Ji, B.G. Thomas, and S.P. Vanka: Metall. Mater. Trans. B, 2011, vol. 42B, pp. 987-1007.

10. D. Schurmann, I. Glavinić, B. Willers, K. Timmel, and S. Eckert: Metall. Mater. Trans. B, 2020, vol. 51B, pp. 61-78.

11. K. Timmel, C. Kratzsch, A. Asad, D. Schurmann, R. Schwarze, and S. Eckert: IOP Conf. Ser., 2017, vol. 228, art. no. 012019https://doi.org/10.1088/1757-899X/228/1/012019.

12. B.G. Thomas, R. Singh, S.P. Vanka, K. Timmel, S. Eckert, and G.J. Gerbeth: Manuf. Sci. Prod., 2015, vol. 15 (1), pp. 93-104.

13. D. Schurmann, B. Willers, G. Hackl, Y. Tang, and S. Eckert: Metall. Mater. Trans. B, 2019, vol. 45B, pp. 1024-37.

14. B.G. Thomas: Steel Res. Int., 2018, vol. 89, p. 1700312. https:// doi.org/10.1002/srin.201700312.

15. X. Miao, K. Timmel, D. Lucas, Z. Ren, and S. Eckert: Metall. Mater. Trans. B, 2012, vol. 43B, pp. 954-72.

16. Z. Liu, L. Li, and B. Li: JOM, 2016, vol. 68, pp. 2180-90.

17. Z. Liu, A. Vakhrushev, M. Wu, E. Karimi-Sibaki, A. Kharicha, A. Ludwig, and B. Li: Metals, 2018, vol. 8, p. 609. https://doi.org/ 10.3390/met8080609.

18. S. Cho and B.G. Thomas: JOM, 2020, vol. 72, pp. 3610-27. https://doi.org/10.1007/s11837-020-04329-8.

19. A. Vakhrushev, A. Kharicha, Z. Liu, M. Wu, A. Ludwig, G. Nitzl, Y. Tang, G. Hackl, and J. Watzinger: Metall. Mater. Trans. B, 2020, vol. 51B, pp. 2811-28. https://doi.org/10.1007/s11663-020-01952-3.

20. Z. Li, L. Zhang, D. Ma, and E. Wang: Metall. Mater. Trans. B, 2020, vol. 51B, pp. 2609-27. https://doi.org/10.1007/s11663-02001950-5. 
21. S. Garcia-Hernandez, C.H. Gonzalez-Guzman, R. Morales Davila, J.J. Barreto, E. Gutierrez, and I. Calderon-Ramos: Crystals, 2020, vol. 10 (11), p. 958. https://doi.org/10.3390/cryst10110958.

22. A. Vakhrushev, A. Kharicha, M. Wu, A. Ludwig, G. Nitzl, Y. Tang, G. Hackl, J. Watzinger, and C.M.G. Rodrigues: IOP Conf. Ser., 2020, vol. 861, art. no. 012015https://doi.org/10.1088/1757$899 \mathrm{X} / 861 / 1 / 012015$.

23. H.G. Weller, G. Tabor, H. Jasak, and C. Fureby: Comput. Phys., 1998, vol. 12 (6), pp. 620-31.

24. F. Nicoud and F. Ducros: Flow Turbul. Combust., 1999, vol. 63 (3), pp. 183-200.

25. J. Smagorinsky: Mon. Weather Rev., 1963, vol. 91, pp. 99-164.

26. A. Vakhrushev, Z. Liu, M. Wu, A. Kharicha, A. Ludwig, G. Nitzl, Y. Tang, and G. Hackl: Numerical Modelling of the MHD Flow in Continuous Casting Mold by Two CFD Platforms ANSYS Fluent and OpenFOAM, 7th Int. Congr. on Science and Technology of Steelmaking (ICS 2018), Venice, Italy, 2018, p. 159

27. H. Kobayashi: Phys Fluids., 2006, vol. 18, art. no. 045107https:// doi.org/10.1063/1.2194967.

28. P.A. Davidson: Introduction to Magnetohydrodynamics, Cambridge University Press, Cambridge, 2001.
29. Y. Plevachuk, V. Sklyarchuk, S. Eckert, G. Gerbeth, and R. Novakovic: J. Chem. Eng. Data, 2014, vol. 59, pp. 757-63.

30. A. Ribes and C. Caremoli: Salomé Platform Component Model for Numerical Simulation, COMPSAC 07: Proc. 31st Annual Int. Computer Software and Applications Conf., IEEE Computer Society, Washington, DC, 2007, pp. 553-64, https://doi.org/10.1 109/COMPSAC.2007.185.

31. E. Mas de les Valls: Tesi Doctoral, Departament de Física i Enginyeria Nuclear, Universitat Politècnica de Catalunya, http:// hdl.handle.net $/ 2117 / 95157$.

32. J.A. Shercliff: Math. Proc. Cambridge Philos. Soc., 1953, vol. 49 (1), pp. 136-44.

33. J. Sommeria and R. Moreau: J. Fluid Mech., 1982, vol. 118, pp. $507-18$.

34. S. Smolentsev: Theor. Comput. Fluid Dyn., 2009, vol. 23, pp. 557-70.

35. J.C.R. Hunt, A.A. Wray, and P. Moin: Center for Turbulence Research Report CTR-S88, 1988, pp. 193-208.

36. V. Kolář: Int. J. Heat Fluid Flow, 2007, vol. 28 (4), pp. 638-52.

Publisher's Note Springer Nature remains neutral with regard to jurisdictional claims in published maps and institutional affiliations. 